OPEN ACCESS

Edited by:

Xihui Shen,

Northwest $A$ and F University, China

Reviewed by:

Emilio G. Vozza,

Trinity College Dublin, Ireland

Tamaki Yano,

Tohoku University, Japan

Pedro Escoll,

Institut Pasteur, France

*Correspondence:

Hongbing Han

hanhongbing@cau.edu.cn

${ }^{t}$ These authors have contributed equally to this work

Specialty section:

This article was submitted to

Bacteria and Host,

a section of the journal

Frontiers in Cellular and

Infection Microbiology

Received: 30 July 2021 Accepted: 23 September 2021 Published: 07 October 2021

Citation:

Wang M, Fan Z and

Han H (2021) Autophagy in Staphylococcus aureus Infection. Front. Cell. Infect. Microbiol. 11:750222. doi: 10.3389/fcimb.2021.750222

\section{Autophagy in Staphylococcus aureus Infection}

\author{
Mengyao Wang ${ }^{1,2,3+}$, Ziyao Fan $^{4 \dagger}$ and Hongbing Han ${ }^{1,2,3 *}$ \\ ${ }^{1}$ Beijing Key Laboratory of Animal Genetic Improvement, College of Animal Science and Technology, China Agricultural \\ University, Beijing, China, ${ }^{2}$ National Engineering Laboratory for Animal Breeding, College of Animal Science and Technology, \\ China Agricultural University, Beijing, China, ${ }^{3}$ Key Laboratory of Animal Genetics, Breeding and Reproduction of the Ministry \\ of Agriculture and Rural Affairs, College of Animal Science and Technology, China Agricultural University, Beijing, China, \\ ${ }^{4}$ Genome Analysis Laboratory of the Ministry of Agriculture and Rural Affairs, Agricultural Genomics Institute at Shenzhen, \\ Chinese Academy of Agricultural Sciences, Shenzhen, China
}

Staphylococcus aureus is an invasive, facultative intracellular pathogen that can colonize niches in various host organisms, making it difficult for the host immune system to completely eliminate. Host autophagy is an intracellular clearance pathway involved in degrading $S$. aureus. Whereas the accessory gene regulatory system of $S$. aureus that controls virulence factors could resist the host immune defenses by evading and even utilizing autophagy. This article reviews the interaction between autophagy and S. aureus, providing insights on how to use these mechanisms to improve $S$. aureus infection control.

Keywords: Staphylococcus aureus, autophagy, accessory gene regulatory system, intracellular persistence, hostpathogen interactions

\section{INTRODUCTION}

Staphylococcus aureus is an opportunistic pathogen that has adapted to long-term colonization in the human skin and nares (Jeon et al., 2020). S. aureus utilizes the adhesins to initiate the invasion process by attaching to the surface of host cell (Horn et al., 2018; Watkins and Unnikrishnan, 2020). After invasion, S. aureus induces a cytoplasmic and mitochondrial $\mathrm{Ca}^{2+}$ overload, which leads to both apoptotic and necrotic cell death (Stelzner et al., 2020). S. aureus infection presents as longlasting persistent or acute diseases that are associated with significant morbidity and mortality (Turner et al., 2019). Antibiotics were most widely used to treat $S$. aureus infectious diseases, however, S. aureus has rapidly developed resistance to antibiotics. Approximately $90 \%$ of $S$. aureus strains show resistance to multiple antibiotics, resulting in decreased antibiotic application and reduced antibiotic effectiveness (Costa et al., 2018). Since methicillin-resistant S. aureus (MRSA) was identified in 1960, the infection rate with MRSA has increased globally. It leads to an increased burden on healthcare-associated expenditures (Zhen et al., 2020), and has become the main cause of bacterial infection in hospitals and communities (Lakhundi and Zhang, 2018). MRSA strains account for 5\%-82\% of S. aureus isolates (Köck et al., 2010; Falagas et al., 2013), leading to clinical syndromes including bacteremia (Klevens et al., 2007), one of the most severe situations of S. aureus infections with 15\%-60\% mortality rates (Li et al., 2021). Invasive MRSA strains possess a series of virulence factors and toxins, allowing them to spread rapidly in the community, and seriously 
threaten public health (Lakhundi et al., 2018). Therefore, new strategies to control $S$. aureus infection have gradually become the focus by manipulating and enhancing host immune defenses (Keller et al., 2020; Gauron et al., 2021).

The host immune system provides the first defense against pathogens, effectively removing intracellular pathogens in most cases. Simultaneously, autophagy also plays an essential role in resisting to pathogens (Randow et al., 2013). Autophagy is a fundamental biological process, in which pathogens are engulfed by double membrane vesicles called phagophores and eventually transported to lysosomes for subsequent degradation (Kirkegaard et al., 2004; Nakatogawa, 2020). Recent studies have demonstrated that autophagy has a crucial role in host cell defense against $S$. aureus (Lv et al., 2019; Gibson et al., 2020). The cell wall components of $S$. aureus can be detected as pathogen-associated molecular patterns (PAMPs) and then induce autophagy (Arroyo et al., 2013; Wu et al., 2016). Autophagy effectively limits S. aureus growth by fusion with the lysosome or positively regulating the phagocytosis of macrophages (Lv et al., 2019; Gibson et al., 2020). Researchers have thus tried to use the autophagy pathway to control $S$. aureus infection. Whereas some $S$. aureus strains have evolved self-defense mechanisms against autophagy degradation, and are even protected by the autophagy pathway (Schnaith et al., 2007). Once $S$. aureus enters the autophagosome, it transforms this "compartment" to create a hospitable environment in which it can survive and replicate (O'Keeffe et al., 2015). S. aureus being degraded by the autophagy pathway or protected by the autophagosome compartment is related to the accessory gene regulatory (agr) system which plays a crucial role in pathogenesis by coordinating virulence factors expression and bacterial density (Schnaith et al., 2007; O'Keeffe et al., 2015).

Here, we reviewed "beneficial" and "harmful" functions of autophagy in the process of $S$. aureus infection, as well as the mechanism by which $S$. aureus evades autophagy. This review is helpful to understand the interaction between hosts and $S$. aureus, and provides a theoretical basis for the development of new treatments for $S$. aureus infection.

\section{S. AUREUS CAN INFECT HOST AS A FACULTATIVE INTRACELLULAR PATHOGEN}

Based on phylogenetic analyses, Queck et al. reported that S. aureus first emerged as a nonvirulent species, and only later acquired virulent functions (Queck et al., 2008). The agr quorum sensing system is the main virulence regulator of $S$. aureus in response to changing environmental conditions, such as adapting to lownutrition conditions in high-cell-density populations, forming a nonpathogenic lifestyle (Queck et al., 2008). Approximately 30\% of humans persistently but asymptomatically carry $S$. aureus in their nasopharynx (Wertheim et al., 2005). S. aureus actively adheres to promote colonization and replicates to avoid removal by nasal secretions (Foster et al., 2014).

The cell wall-anchored proteins of $S$. aureus, Fnbps and IsdB, promote internalization and subsequent invasion (Zapotoczna et al., 2013; Schlesier et al., 2020). The pattern recognition receptors (PRRs) expressed on the surface of phagocytes recognize pathogens and mediate their uptake into phagosomes for later elimination (Flannagan et al., 2009). Nonprofessional phagocytes utilize endocytosis to take up S. aureus (Moldovan and Fraunholz, 2019). Once internalized by host cells, the agr system of $S$. aureus increases virulence factors to damage phagosomes and promote intracellular survival (Novick et al., 1993). The phagosome or endosome can fuse directly with a lysosome to acidify to low $\mathrm{pH}$ for degrading microorganisms (Flannagan et al., 2009; Lâm et al., 2010). However, S. aureus tolerates acidic environments, which contributes to its survival within phagolysosomes (Weinrick et al., 2004). Exposure to an acidic environment increased expression of agr system (Tranchemontagne et al., 2016). Phagosomal acidification even appears to be essential for survival of some $S$. aureus strains (Tranchemontagne et al., 2016). Agr positively regulates cytotoxic phenol-soluble modulins (PSMs), which mediate escape from the phagosome into the cytoplasm to avoid lysosomal killing (Grosz et al., 2014; Münzenmayer et al., 2016). The cytoplasmically located $S$. aureus or leaky phagosomes could be captured by autophagosomal membranes and eventually fuse with lysosomes for autophagic degradation (Fraunholz and Sinha, 2012). $S$. aureus is also capable of escaping or even manipulating the autophagy pathway for replication and dissemination (Vozza et al., 2021). S. aureus further evolved regulatory functions to attenuate the expression of virulence genes to reduce innate immune defenses (Boisset et al., 2007; Cheung et al., 2014). This decreases the proinflammatory potential of $S$. aureus, which is associated with chronic infection. Surprisingly, S. aureus is very responsive to external stimuli, and rapidly reverts back to the original virulent state in rich bacterial growth conditions (Tuchscherr et al., 2020).

\section{THE EFFECT OF AUTOPHAGY ON INTRACELLULAR S. AUREUS}

Autophagy is considered a crucial intracellular degradation system for removing dangerous pathogens (Levine, 2005). The dynamic membrane processes of autophagy occur through regulators comprised of autophagy-related genes (ATGs) and additional factors based on the following sequential steps: autophagy initiation; phagophore formation; double-membrane nucleation and phagophore elongation; cytoplasmic microorganism engulfment; autophagosome fusion with lysosome; and cargo degradation (Kuo et al., 2018) (Figure 1).

\section{Manipulation of Autophagy on S. aureus in Non-Professional Phagocytes}

In the non-professional phagocytes, intracellular S. aureus is rapidly ubiquitinated and then recognized by autophagy receptors, including sequestosome 1 (SQSTM1/p62), nuclear domain protein 52 (NDP52/CALCOCO2), and optineurin (OPTN) (Neumann et al., 2016) (Figure 1). These receptors characteristically couple ubiquitin to microbes with the autophagosomal membrane-associated protein LC3, to trap bacteria in autophagosomes (Mestre et al., 2010; Neumann 


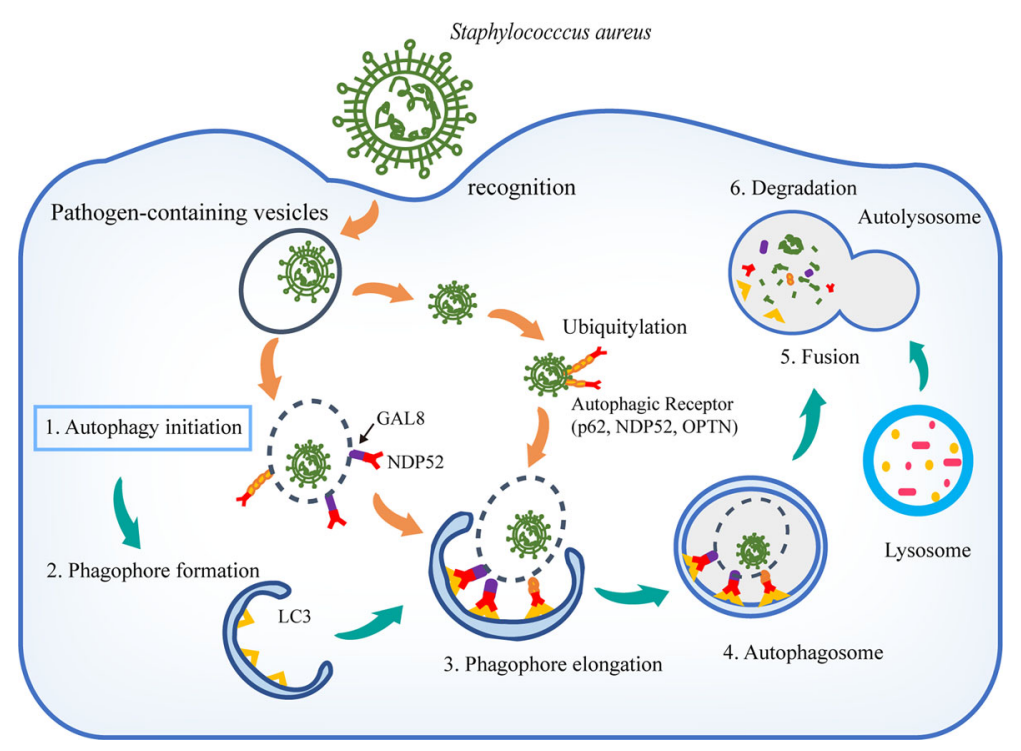

FIGURE 1 | Host autophagy in defense against S. aureus. The components of S. aureus are detected as PAMPs by host PRRs, and autophagy is induced. Autophagy receptors p62, NDP52, and OPTN function as bridging adaptors to induce selective autophagic degradation of invading pathogens by specifically recognizing ubiquitin-coated intracellular pathogens. Damaged pathogen-containing vesicles are detected by GAL8. GAL8 monitors endosomal integrity and activates antibacterial autophagy in conjunction with the autophagy receptor NDP52. During autophagy, LC3 is recruited to autophagosomal membranes. Autophagosome subsequently fuses with a lysosome to form the autolysosome, where the acidic environment and enzymes mediate the bacterial degradation.

et al., 2016). Endosomes that are damaged by S. aureus are targeted by Galectin-8 (GAL8) to attract autophagosomal clearance (Soong et al., 2015). Phospholipase C-related catalytically inactive protein (PRIP) has been shown to be required for the autophagosome maturation and acidification, which facilitates the $S$. aureus elimination by promoting the fusion of $S$. aureus-containing autophagosomes with lysosomes in mouse embryonic fibroblasts (Harada-Hada et al., 2014). Recently, the positive role of autophagy was further supported by autophagy protein which mediates a novel form of defense in response to $S$. aureus infection. ATG16L1 protects host cells from $S$. aureus by releasing ADAM10 (a disintegrin and metalloproteinase 10) as a bacterial toxin scavenger in alveolar epithelial cells. Loss of ATG16L1 expression exacerbates $S$. aureus-induced mortality in mice (Becker et al., 2014; Keller et al., 2020). Except for the above resistance mechanisms that reduce $S$. aureus burden, autophagy could protect host cells against $S$. aureus infection by maintaining tolerance toward the pore forming alpha-toxin ( $\alpha$-toxin) secreted by $S$. aureus (Maurer et al., 2015). Increased cell death induced by $\alpha$-toxin was observed in mouse endothelial cells upon autophagy inhibition, revealing that autophagy was a barrier of cells to maintain membrane homeostasis under stress conditions (Maurer et al., 2015).

However, S. aureus has developed mechanisms to escape from the autophagy pathway (Riebisch et al., 2021). It has been demonstrated that $S$. aureus can block autophagosome maturation via phosphorylation of mitogen-activated protein kinase 14 (MAPK14) and ATG5 in murine fibroblasts (Neumann et al., 2016). S. aureus secreted $\alpha$-toxin was shown to inhibit the fusion of autophagosomes with lysosomes to prevent $S$. aureus degradation before reaching the cytoplasm (Mestre et al., 2010). The $S$. aureus-containing autophagosomes neither acidified nor acquired lysosome-associated membrane protein-2 (LAMP-2), a marker for late endosomes and lysosomes. This dysfunctional autophagic response was also observed in S. aureus infected bovine mammary epithelial cells (Wang et al., 2019). After escape from autophagosomes, $S$. aureus proliferates extensively in the cytoplasm and eventually results in the lysis of host cell (Schnaith et al., 2007). In addition to avoiding autophagy, some S. aureus have developed to utilize autophagy for their own benefit in host cells. $\mathrm{Na}$ Geng et al. described that $S$. aureus caused obvious induction of autophagosomes formation to facilitate intracellular replication in bovine mammary epithelial cells (Geng et al., 2020). It was also supported by a recent study that autophagy suppressed by overexpression of protein kinase C (PKC) could inhibit $S$. aureus intracellular replication in Chinese hamster ovary cells (Gauron et al., 2021). Additionally, Bravo-Santano et al. demonstrated glucose and amino acid pools were severely depleted by $S$. aureus to induce a starvation response, which leads to highly activated glutamine in host cells for their own metabolic needs. These changes activate autophagy through AMP-activated protein kinase (AMPK) and extracellular signal-regulated kinase (ERK) signaling pathways. Metabolic activation of autophagy is used by $S$. aureus to sustain its own intracellular survival (Bravo-Santano et al., 2018).

\section{The Effect of Autophagy in S. aureus Infected Professional Phagocytes}

In professional phagocytes, phagocytosed $S$. aureus are initially located in a phagocytic vesicle. The vacuolar pathogens can be 


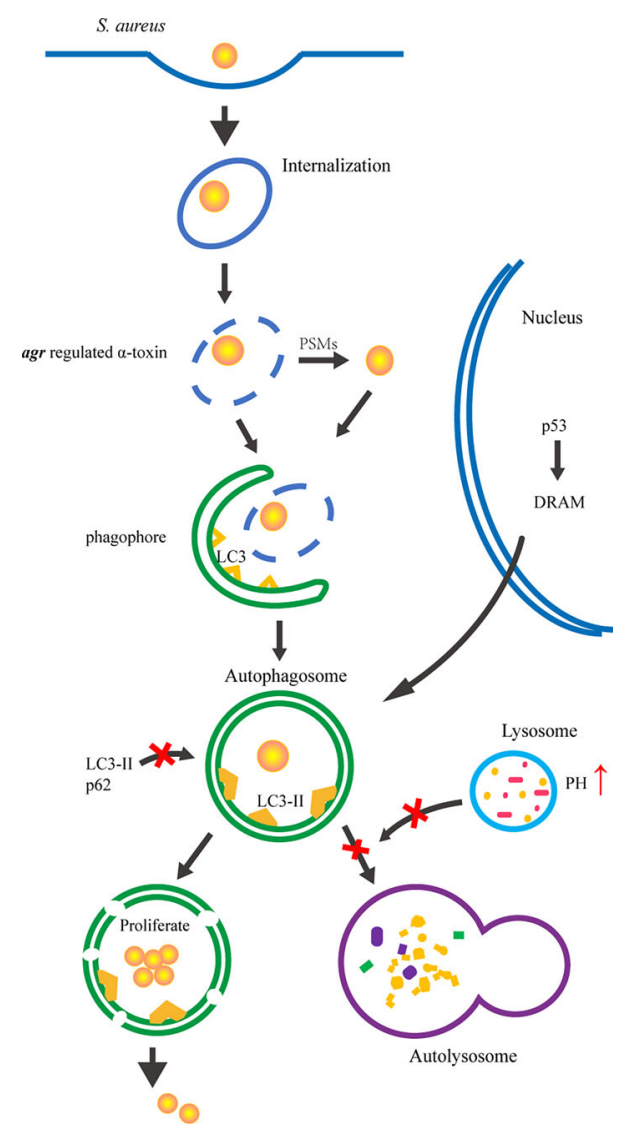

FIGURE 2 | The interaction between autophagy and agr locus of S. aureus. S. aureus is internalized into host cell via the endocytic pathway. Agr system positively regulates $\alpha$-toxin and PSMs to mediate escape from the phagosome into cytoplasm. The escaped S. aureus and damaged phagosomes are engulfed by phagophores. The expression of LC3-II and p62 are blocked by agr system to hinder autophagosomal maturation. Upon autophagosomal maturation, agr as the pH sensitive system inhibit the fusion of autophagosome and lysosome to escape autophagic degradation. Thus, the autophagosomes provide a niche for S. aureus replication. In addition, agr-specific factor was found to accumulate autophagosomes as intracellular survival niches by manipulating the p53/DRAM pathway in human PMNs, whereas has not been found in other species or cell types. Eventually, S. aureus escape from autophagosomes into the cytoplasm and induces host cell death.

sequestered into autophagic membranes to thereby eventually fuse with lysosomes (Knodler and Celli, 2011). The autophagy receptor SQSTM1/p62 has been shown to directly co-localize with $S$. aureus in the cytosol in neutrophils for autophagic degradation. SQSTM1/ p62 knockdown significantly impaired host defense and increased susceptibility of neutrophils to S. aureus (Gibson et al., 2020). Besides being an autophagy receptor, SQSTM1/p62 brings the precursor protein of ribosomal protein S30 and additional ubiquitinated protein complexes to autolysosomes, where they were processed from innocuous forms into bactericidal products (Ponpuak et al., 2010). Thus, SQSTM1/p62 is crucial in antibacterial action in host cells. Autophagy also controls S. aureus infection by promoting phagocytosis in macrophages. Decreased level of autophagy through the PI3K inhibitor LY294002 or knockdown of Beclin1 treatment significantly weakens phagocytosis of $S$. aureus-infected macrophages, indicating that $S$. aureus-induced autophagy contributes to the phagocytosis of macrophages ( $\mathrm{Lv}$ et al., 2019). Moreover, the intracellular autophagy-related molecule microtubule-associated protein $1 \mathrm{~S}$ (MAP1S) promotes phagocytosis of $S$. aureus by enhancing the MyD88-dependent TLR signaling pathway. The Map1S-deficient macrophages exhibit impaired S. aureus phagocytosis (Shi et al., 2016). These lines of evidence demonstrate autophagy has a crucial role in eliminating S. aureus.

By contrast, this cellular defense program has also been identified as providing a niche for intracellular S. aureus replication. Some studies reported S. aureus are protected from degradation within autophagosomes of phagocytes, and have obtained an intracellular survival niche, which ultimately facilitates dissemination in the host (O'Keeffe et al., 2015; Mulcahy et al., 2020). S. aureus escapes autophagic degradation by blocking autophagy flux (LC3-II, p62) and increasing the $\mathrm{pH}$ in autolysosomes after invading macrophages (Cai et al., 2020). It has been reported chemical inhibition of the autophagic response by 3 methyladenine (3-MA) promoted phagocytosis of mouse macrophages (Zhu et al., 2018) and prevented the escape of $S$. aureus in mouse bone marrow-derived dendritic cells (O'Keeffe et al., 2015). These data indicate that inhibiting the formation of 
autophagosomes facilitates elimination intracellular S. aureus. S. aureus also have developed to utilize autophagy in professional phagocytes. In primary human polymorphonuclear neutrophils (PMNs), S. aureus enhances the accumulation of autophagosomes in cells by activating the stress response pathway to maintain the survival niche (Mulcahy et al., 2020). At the meantime, S. aureus could disrupt the apoptotic pathway of PMNs to prevent the destruction of its intracellular niche and protect itself from subsequent macrophages phagocytosis (Vozza et al., 2021). The non-canonical form of autophagy machinery LC3-associated phagocytosis (LAP), which is dependent on NADPH oxidase, can also be utilized by intracellular $S$. aureus for pathogenesis. At the early stage of infection in zebrafish neutrophils, the autophagy marker LC3 rapidly decorates $S$. aureus-containing singlemembrane phagosomes. The formation of LC3-positive and nonacidified phagosomes provide a spacious area for $S$. aureus to safely replicate (Prajsnar et al., 2020).

\section{THE EFFECT OF AGR ON AUTOPHAGY CONTROLLING INTRACELLULAR S. AUREUS}

Agr system is a major gene regulator that governs the toxin production of $S$. aureus at the appropriate time, regulating the adhesins expression during attachment and virulence factors during infection. Agr can upregulate $\alpha$-toxin to cause tissue destruction by perturbing to epithelial cell junctions (von Hoven and Husmann, 2019). $\alpha$-toxin also increased S. aureus internalization within mast cells by up-regulation of $\beta 1$ integrin (Goldmann et al., 2016). After internalization, the high-level expression of agr led to strong expression of toxins and exoenzymes, as well as increased expression of methicillin resistance genes, mediating the pathogenesis (Cheung et al., 2011). At the meantime, the agr locus controlled phenol-soluble modulins alpha (PSM $\alpha$ ) has also been shown to be crucial for phagosomal escape in both professional and non-professional phagocytes (Grosz et al., 2014). When agr is absent, phagosomal escape and autophagosomal accumulation are significantly reduced as well as intracellular bacterial burden is reduced (O'Keeffe et al., 2015; Blättner et al., 2016). Additionally, agr has been shown to have the alternating function, which can reduce cytotoxicity to survive persistently within host cells and avoid the host immune system activation (Tuchscherr et al., 2011).

Schnaith et al. reported agr-regulated factor(s) activated autophagy could prevent the maturation of $S$. aureus-containing phagosomes in human epithelial cells (Schnaith et al., 2007). Subsequently, the agr regulated $\alpha$-toxin was shown to be necessary for eliciting autophagy, but the autophagic response was dysfunctional and the induced autophagosomes were not acidic. Additionally, $\alpha$-toxin-deficient $S$. aureus strains were unable to activate the autophagy pathway (Mestre et al., 2010) (Figure 2). In addition, an agr-specific factor was discovered that manipulates the autophagy network to provide an intracellular niche for S. aureus in human PMNs, but whether it is $\alpha$-toxin has yet to be determined. The normal autophagic flux, expression of LC3II and p62, was disrupted in PMNs containing S. aureus (Mulcahy et al., 2020). Agr-positive S. aureus leads to the accumulation of autophagy inducer p53 in PMNs, driving transcriptional activation of pro-autophagic membrane protein damage-regulated autophagy monitor (DRAM). DRAM can directly mediate p53-induced autophagy and enhance the accumulation of autophagosomes in cells in order to maintain a survival niche for S. aureus. Within these induced autophagosomes, $S$. aureus are protected and ultimately facilitates dissemination. $S$. aureus survival rate is significantly reduced using an agr-deficient mutant, suggesting that the agr locus is crucial for autophagy-mediated intracellular survival (Mulcahy et al., 2020). Similarly, the agr mutant showed a significantly reduced intracellular survival rate in mouse phagocytes because they fail to accumulate $\mathrm{LC} 3-\mathrm{II}^{+}$ autophagosomes and are delivered efficiently to lysosomes (O'Keeffe et al., 2015). These results indicate that agr-regulated factors determined the ability of $S$. aureus for autophagy targeting and avoidance of lysosomal degradation in host cells (Figure 2). However, in human osteosarcoma cells, agr-positive S. aureus strains were more efficiently entrapped in autophagosomes than agr-negative $S$. aureus (Mauthe et al., 2012). Additionally, a recently study showed the absence of agr regulated PSMs increased $S$. aureus long-term survival in human endothelial cells (Siegmund et al., 2021). Thus, a comprehensive analysis of different $S$. aureus strains as well as various cell types is required to elucidate the interplay between agr and autophagy.

\section{SUMMARY AND PROSPECT}

The emergence of antibiotic-resistant strains of bacteria requires the continuous development of new antibiotics. However, drug development is a very long and expensive process. The exploration of new drugs for effective treatment of $S$. aureus infection is a difficult task. Many years of research have produced a few drugs, such as penicillin, vancomycin, and cephalosporin. However, once the pathogen becomes intracellular, antibiotics will not achieve the expected effect, and new antibiotics may have an impact on cell function. In this case, development of new molecules must be explored in order to defeat $S$. aureus. Autophagy, as an innate immune response mechanism, degrades $S$. aureus in cells. Strains with high agr activity are usually able to escape and replicate intracellularly using autophagy, while strains lacking agr systems are usually unable to escape the autophagosomes and are eventually degraded.

Appropriate doses of the autophagy modulators could be an effective strategy for controlling infection. A recent study has reported the natural coumarin derivative daphnetin (DAPH) effectively enhances autophagic pathway to exert an antibacterial effect against S. aureus (Zhang et al., 2019). Moreover, selenium has been shown to inhibit the proliferation of $S$. aureus by promoting autophagy pathway in $S$. aureus infected mouse macrophages (Zang et al., 2020). Regardless of S. aureus strain identity, their methods of escaping autophagy pathway usually involve blocking autophagy flux. The recently developed $\mathrm{pH}$ responsive polymersome (Xu et al., 2020) loaded with LC3 and p62, disintegrates after encountering lysosomes with low $\mathrm{pH}$, releasing the loaded proteins to supplement autophagy flux, 
which could be a new strategy. However, the situation is more complicated than expected, the fact that autophagy inducers seem to be beneficial for treating $S$. aureus infections, but in turn might facilitate other bacterial infections (Escoll et al., 2016). Therefore, the use of autophagy modulators should be highly cautious. Additionally, the ability of $S$. aureus to escape and survive in the cytosol are dependent on both the strain and cell type. Treatment with autophagy inhibitors was shown to reduce $S$. aureus load, and the autophagy induction by rapamycin restored replication of $S$. aureus (Schnaith et al., 2007; BravoSantano et al., 2018). It is difficult to perform corresponding treatment of $S$. aureus infections with different genetic backgrounds and different targeted specific cell types.

We need novel approaches to suppress intracellular S. aureus load with minimal side effect to the host. And obviously, the significance of eliminating intracellular bacteria for effective treatment of persistent $S$. aureus infections has received more attention. The vancomycin encapsulated within liposomes was shown to be taken up efficiently by Kupffer cells and killed intracellular $S$. aureus, which reduced the mortality of mice, whereas free vancomycin could not (Surewaard et al., 2016). Combining autophagy modulators with the liposomes may be a promising strategy. The recent focus on developing strategies for intracellular $S$. aureus is encouraging and may lead to more effective treatments in the near future.

\section{AUTHOR CONTRIBUTIONS}

MW performed the literature survey and wrote the draft. ZF critically reviewed and improved the manuscript. $\mathrm{HH}$ contributed to critical evaluation and finalizing of the review. All authors contributed to the article and approved the submitted version.

\section{FUNDING}

This study was supported by the National Key R\&D Program of Intergovernmental Key Projects in China (2018YFE0101700).

\section{REFERENCES}

Arroyo, D. S., Soria, J. A., Gaviglio, E. A., Garcia-Keller, C., Cancela, L. M., Rodriguez-Galan, M. C., et al. (2013). Toll-Like Receptor 2 Ligands Promote Microglial Cell Death by Inducing Autophagy. FASEB J. Off. Publ. Fed. Am. Soc. Exp. Biol. 271, 299-312.

Becker, R. E., Berube, B. J., Sampedro, G. R., DeDent, A. C., and Bubeck Wardenburg, J. (2014). Tissue-Specific Patterning of Host Innate Immune Responses by Staphylococcus Aureus $\alpha$-Toxin. J. Innate Immun. 65, 619-631. doi: 10.1159/000360006

Blättner, S., Das, S., Paprotka, K., Eilers, U., Krischke, M., Kretschmer, D., et al. (2016). Staphylococcus Aureus Exploits a Non-Ribosomal Cyclic Dipeptide to Modulate Survival Within Epithelial Cells and Phagocytes. PloS Pathog. 129, e1005857. doi: 10.1371/journal.ppat.1005857

Boisset, S., Geissmann, T., Huntzinger, E., Fechter, P., Bendridi, N., Possedko, M., et al. (2007). Staphylococcus Aureus RNAIII Coordinately Represses the Synthesis of Virulence Factors and the Transcription Regulator Rot by an Antisense Mechanism. Genes Dev. 2111, 1353-1366. doi: 10.1101/gad.423507

Bravo-Santano, N., Ellis, J. K., Mateos, L. M., Calle, Y., Keun, H. C., Behrends, V., et al. (2018). Intracellular Staphylococcus Aureus Modulates Host Central Carbon Metabolism To Activate Autophagy. mSphere 34. doi: 10.1128/ mSphere.00374-18

Cai, J., Li, J., Zhou, Y., Wang, J., Li, J., Cui, L., et al. (2020). Staphylococcus Aureus Facilitates Survival in Bovine Macrophages by Blocking Autophagic Flux. J. Cell. Mol. Med. 246, 3460-3468. doi: 10.1111/jcmm.15027

Cheung, G. Y., Kretschmer, D., Duong, A. C., et al. (2014). Production of an Attenuated Phenol-Soluble Modulin Variant Unique to the MRSA Clonal Complex 30 Increases Severity of Bloodstream Infection. PloS Pathog. 108, e1004298. doi: 10.1371/journal.ppat.1004298

Cheung, G. Y., Wang, R., Khan, B. A., Sturdevant, D. E., and Otto, M. (2011). Role of the Accessory Gene Regulator Agr in Community-Associated MethicillinResistant Staphylococcus Aureus Pathogenesis. Infect. Immun. 795, 19271935. doi: 10.1128/IAI.00046-11

Costa, F. N., Belo, N. O., Costa, E. A., Andrade, G. I., Pereira, L. S., Carvalho, I. A., et al. (2018). Frequency of Enterotoxins, Toxic Shock Syndrome Toxin-1, and Biofilm Formation Genes in Staphylococcus Aureus Isolates From Cows With Mastitis in the Northeast of Brazil. Trop. Anim. Health Prod. 505, 1089-1097. doi: 10.1007/s11250-018-1534-6

Escoll, P., Rolando, M., and Buchrieser, C. (2016). Modulation of Host Autophagy During Bacterial Infection: Sabotaging Host Munitions for Pathogen Nutrition. Front. Immunol. 7, 81. doi: 10.3389/fimmu.2016.00081

Falagas, M. E., Karageorgopoulos, D. E., Leptidis, J., and Korbila, I. P. (2013) MRSA in Africa: Filling the Global Map of Antimicrobial Resistance. PloS One 87, e68024. doi: 10.1371/journal.pone.0068024

Flannagan, R. S., Cosío, G., and Grinstein, S. (2009). Antimicrobial Mechanisms of Phagocytes and Bacterial Evasion Strategies. Nat. Rev. Microbiol. 75, 355-366. doi: $10.1038 /$ nrmicro2128

Foster, T. J., Geoghegan, J. A., Ganesh, V. K., and Höök, M. (2014). Adhesion, Invasion and Evasion: The Many Functions of the Surface Proteins of Staphylococcus Aureus. Nat. Rev. Microbiol. 121, 49-62. doi: 10.1038/ nrmicro3161

Fraunholz, M., and Sinha, B. (2012). Intracellular Staphylococcus Aureus: Live-in and Let Die. Front. Cell. Infect. Microbiol. 2, 43. doi: 10.3389/fcimb.2012.00043

Gauron, M. C., Newton, A. C., and Colombo, M. I. (2021). Pkc $\alpha$ Is Recruited to Staphylococcus Aureus-Containing Phagosomes and Impairs Bacterial Replication by Inhibition of Autophagy. Front. Immunol. 12, 662987. doi: 10.3389/fimmu.2021.662987

Geng, N., Liu, K., Lu, J., Xu, Y., Wang, X., Wang, R., et al. (2020). Autophagy of Bovine Mammary Epithelial Cell Induced by Intracellular Staphylococcus Aureus. J. Microbiol. (Seoul Korea) 584, 320-329. doi: 10.1007/s12275-0209182-8

Gibson, J. F., Prajsnar, T. K., Hill, C. J., Tooke, A. K., Serba, J. J., Tonge, R.D., et al. (2020). Neutrophils Use Selective Autophagy Receptor Sqstm1/p62 to Target Staphylococcus Aureus for Degradation In Vivo in Zebrafish. Autophagy 1-10. doi: 10.1080/15548627.2020.1765521

Goldmann, O., Tuchscherr, L., Rohde, M., and Medina, E. (2016). $\alpha$-Hemolysin Enhances Staphylococcus aureus Internalization and Survival Within Mast Cells by Modulating the Expression of $\beta 1$ Integrin. Cellular Microbiol. 186, 807-819. doi: $10.1111 / \mathrm{cmi} .12550$

Grosz, M., Kolter, J., Paprotka, K., Winkler, A. C., Schäfer, D., Chatterjee, S. S., et al. (2014). Cytoplasmic Replication of Staphylococcus Aureus Upon Phagosomal Escape Triggered by Phenol-Soluble Modulin $\alpha$. Cell. Microbiol. 164, 451-465. doi: $10.1111 / \mathrm{cmi} .12233$

Harada-Hada, K., Harada, K., Kato, F., Hisatsune, J., Tanida, I., Ogawa, M., et al. (2014). Phospholipase C-Related Catalytically Inactive Protein Participates in the Autophagic Elimination of Staphylococcus Aureus Infecting Mouse Embryonic Fibroblasts. PloS One 95, e98285. doi: 10.1371/journal.pone.0098285

Horn, J., Stelzner, K., Rudel, T., and Fraunholz, M. (2018). Inside Job: Staphylococcus Aureus Host-Pathogen Interactions. Int. J. Med. Microbiol. IJMM 3086, 607-624. doi: 10.1016/j.ijmm.2017.11.009

Jeon, Y. J., Gil, C. H., Won, J., Jo, A., and Kim, H. J. (2020). Symbiotic Microbiome Staphylococcus Aureus From Human Nasal Mucus Modulates IL-33-Mediated 
Type 2 Immune Responses in Allergic Nasal Mucosa. BMC Microbiol. 201, 301. doi: 10.1186/s12866-020-01974-6

Keller, M. D., Ching, K. L., Liang, F. X., Dhabaria, A., Tam, K., Ueberheidere, B. M., et al. (2020). Decoy Exosomes Provide Protection Against Bacterial Toxins. Nature 5797798, 260-264. doi: 10.1038/s41586-020-2066-6

Kirkegaard, K., Taylor, M. P., and Jackson, W. T. (2004). Cellular Autophagy: Surrender, Avoidance and Subversion by Microorganisms. Nat. Rev. Microbiol. 24, 301-314. doi: 10.1038/nrmicro865

Klevens, R. M., Morrison, M. A., Nadle, J., Petit, S., Gershman, K., Ray, S., et al. (2007). Invasive Methicillin-Resistant Staphylococcus Aureus Infections in the United States. Jama 29815, 1763-1771. doi: 10.1001/jama.298.15.1763

Knodler, L. A., and Celli, J. (2011). Eating the Strangers Within: Host Control of Intracellular Bacteria via Xenophagy. Cell. Microbiol. 139, 1319-1327. doi: 10.1111/j.1462-5822.2011.01632.x

Köck, R., Becker, K., Cookson, B., van Gemert-Pijnen, J. E., Harbarth, S., Kluytmans, J. , et al. (2010). Methicillin-Resistant Staphylococcus Aureus (MRSA): Burden of Disease and Control Challenges in Europe. Euro Surveillance Bull. Europeen Sur Les Maladies Transmissibles Eur. Communicable Dis. Bull. 1541, 19688.

Kuo, C. J., Hansen, M., and Troemel, E. (2018). Autophagy and Innate Immunity: Insights From Invertebrate Model Organisms. Autophagy 142, 233-242. doi: $10.1080 / 15548627.2017 .1389824$

Lakhundi, S., and Zhang, K. (2018). Methicillin-Resistant Staphylococcus Aureus: Molecular Characterization, Evolution, and Epidemiology. Clin. Microbiol. Rev. 314, e00020-18.

Lâm, T. T., Giese, B., Chikkaballi, D., Kühn, A., Wolber, W., Pané-Farré, J., et al. (2010). Phagolysosomal Integrity Is Generally Maintained After Staphylococcus Aureus Invasion of Nonprofessional Phagocytes But Is Modulated by Strain 6850. Infect. Immun. 788, 3392-3403.

Levine, B. (2005). Eating Oneself and Uninvited Guests: Autophagy-Related Pathways in Cellular Defense. Cell 1202, 159-162.

Li, Z., Zhuang, H., Wang, G., Wang, H., and Dong, Y. (2021). Prevalence, Predictors, and Mortality of Bloodstream Infections Due to MethicillinResistant Staphylococcus Aureus in Patients With Malignancy: Systemic Review and Meta-Analysis. BMC Infect. Dis. 211, 74. doi: 10.1186/s12879021-05763-y

Lv, Y., Fang, L., Ding, P., and Liu, R. (2019). PI3K/Akt-Beclin1 Signaling Pathway Positively Regulates Phagocytosis and Negatively Mediates NF-Kb-Dependent Inflammation in Staphylococcus Aureus-Infected Macrophages. Biochem. Biophys. Res. Commun. 5102, 284-289. doi: 10.1016/j.bbrc.2019.01.091

Maurer, K., Reyes-Robles, T., Alonzo, F.3rd, Durbin, J., Torres, V. J., and Cadwell, K. (2015). Autophagy Mediates Tolerance to Staphylococcus Aureus AlphaToxin. Cell Host Microbe 174, 429-440. doi: 10.1016/j.chom.2015.03.001

Mauthe, M., Yu, W., Krut, O., Krönke, M., Götz, F., Robenek, H., et al. (2012). WIPI-1 Positive Autophagosome-Like Vesicles Entrap Pathogenic Staphylococcus Aureus for Lysosomal Degradation. Int. J. Cell Biol. 2012, 179207. doi: $10.1155 / 2012 / 179207$

Mestre, M. B., Fader, C. M., Sola, C., and Colombo, M. I. (2010). Alpha-Hemolysin Is Required for the Activation of the Autophagic Pathway in Staphylococcus Aureus-Infected Cells. Autophagy 61, 110-125. doi: 10.4161/auto.6.1.10698

Moldovan, A., and Fraunholz, M. J. (2019). In or Out: Phagosomal Escape of Staphylococcus Aureus. Cell. Microbiol. 213, e12997. doi: 10.1111/cmi.12997

Mulcahy, M. E., O'Brien, E. C., O'Keeffe, K. M., Vozza, E. G., Leddy, N., and McLoughlin, R. M. (2020). Manipulation of Autophagy and Apoptosis Facilitates Intracellular Survival of Staphylococcus Aureus in Human Neutrophils. Front. Immunol. 11, 565545. doi: 10.3389/fimmu.2020.565545

Münzenmayer, L., Geiger, T., Daiber, E., Schulte, B., Autenrieth, S. E., Fraunholz, M., et al. (2016). Influence of Sae-Regulated and Agr-Regulated Factors on the Escape of Staphylococcus Aureus From Human Macrophages. Cell. Microbiol. 188, 1172-1183. doi: $10.1111 / \mathrm{cmi} .12577$

Nakatogawa, H. (2020). Mechanisms Governing Autophagosome Biogenesis. Nat. Rev. Mol. Cell Biol. 218, 439-458. doi: 10.1038/s41580-020-0241-0

Neumann, Y., Bruns, S. A., Rohde, M., Prajsnar, T. K., Foster, S. J., and Schmitz, I. (2016). Intracellular Staphylococcus Aureus Eludes Selective Autophagy by Activating a Host Cell Kinase. Autophagy 1211, 2069-2084. doi: 10.1080/ 15548627.2016 .1226732

Novick, R. P., Ross, H. F., Projan, S. J., Kornblum, J., Kreiswirth, B., and Moghazeh, S. (1993). Synthesis of Staphylococcal Virulence Factors Is
Controlled by a Regulatory RNA Molecule. EMBO J. 12100, 3967-3975. doi: 10.1002/j.1460-2075.1993.tb06074.x

O'Keeffe, K. M., Wilk, M. M., Leech, J. M., Murphy, A. G., Laabei, M., Monk, I. R., et al. (2015). Manipulation of Autophagy in Phagocytes Facilitates Staphylococcus Aureus Bloodstream Infection. Infect. Immun. 839, 34453457. doi: 10.1128/IAI.00358-15

Ponpuak, M., Davis, A. S., Roberts, E. A., Delgado, M. A., Dinkins, C., Zhao, Z., et al. (2010). Delivery of Cytosolic Components by Autophagic Adaptor Protein P62 Endows Autophagosomes With Unique Antimicrobial Properties. Immunity 323, 329-341. doi: 10.1016/j.immuni.2010.02.009

Prajsnar, T. K., Serba, J. J., Dekker, B. M., Gibson, J. F., Masud, S., Fleming, A., et al. (2020). The Autophagic Response to Staphylococcus Aureus Provides an Intracellular Niche in Neutrophils. Autophagy 1-15. doi: 10.1080/ 15548627.2020.1739443

Queck, S. Y., Jameson-Lee, M., Villaruz, A. E., Bach, T. H., Khan, B. A., Sturdevant, D. E., et al. (2008). RNAIII-Independent Target Gene Control by the Agr Quorum-Sensing System: Insight Into the Evolution of Virulence Regulation in Staphylococcus Aureus. Mol. Cell 321, 150-158. doi: 10.1016/ j.molcel.2008.08.005

Randow, F., MacMicking, J. D., and James, L. C. (2013). Cellular Self-Defense: How Cell-Autonomous Immunity Protects Against Pathogens. Sci. (New York NY) 3406133, 701-706. doi: 10.1126/science.1233028

Riebisch, A. K., Mühlen, S., Beer, Y. Y., and Schmitz, I. (2021). Autophagy-A Story of Bacteria Interfering With the Host Cell Degradation Machinery. Pathog. (Basel Switzerland) 102, 110. doi: 10.3390/pathogens 10020110

Schlesier, T., Siegmund, A., Rescher, U., and Heilmann, C. (2020). Characterization of the Atl-Mediated Staphylococcal Internalization Mechanism. Int. J. Med. Microbiol. IJMM 3108, 151463. doi: 10.1016/ j.ijmm.2020.151463

Schnaith, A., Kashkar, H., Leggio, S. A., Addicks, K., Krönke, M., and Krut, O. (2007). Staphylococcus Aureus Subvert Autophagy for Induction of CaspaseIndependent Host Cell Death. J. Biol. Chem. 2824, 2695-2706. doi: 10.1074/ jbc.M609784200

Shi, M., Zhang, Y., Liu, L., Zhang, T., Han, F., Cleveland, J., et al. (2016). MAP1S Protein Regulates the Phagocytosis of Bacteria and Toll-Like Receptor (TLR) Signaling. J. Biol. Chem. 2913, 1243-1250. doi: 10.1074/ jbc.M115.687376

Siegmund, A., Afzal, M. A., Tetzlaff, F., Keinhörster, D., Gratani, F., Paprotka, K., et al. (2021). Intracellular Persistence of Staphylococcus Aureus in Endothelial Cells Is Promoted by the Absence of Phenol-Soluble Modulins. Virulence 121, 1186-1198. doi: 10.1080/21505594.2021.1910455

Soong, G., Paulino, F., Wachtel, S., Parker, D., Wickersham, M., Zhang, D., et al. (2015). Methicillin-Resistant Staphylococcus Aureus Adaptation to Human Keratinocytes. mBio 62. doi: 10.1128/mBio.00289-15

Stelzner, K., Winkler, A. C., Liang, C., et al. (2020). Intracellular Staphylococcus Aureus Perturbs the Host Cell $\mathrm{Ca}(2+)$ Homeostasis To Promote Cell Death. mBio 116. doi: $10.1128 / \mathrm{mBio} .02250-20$

Surewaard, B. G., Deniset, J. F., Zemp, F. J., Amrein, M., Otto, M., Conly, J., et al. (2016). Identification and Treatment of the Staphylococcus Aureus Reservoir In Vivo. J. Exp. Med. 2137, 1141-1151. doi: 10.1084/jem.20160334

Tranchemontagne, Z. R., Camire, R. B., O'Donnell, V. J., Baugh, J., and Burkholder, K. M. (2016). Staphylococcus Aureus Strain USA300 Perturbs Acquisition of Lysosomal Enzymes and Requires Phagosomal Acidification for Survival Inside Macrophages. Infect. Immun. 841, 241-253. doi: 10.1128/ IAI.00704-15

Tuchscherr, L., Löffler, B., and Proctor, R. A. (2020). Persistence of Staphylococcus Aureus: Multiple Metabolic Pathways Impact the Expression of Virulence Factors in Small-Colony Variants (SCVs). Front. Microbiol. 11, 1028. doi: $10.3389 /$ fmicb.2020.01028

Tuchscherr, L., Medina, E., Hussain, M., Völker, W., Heitmann, V., Niemann, S., et al. (2011). Staphylococcus Aureus Phenotype Switching: An Effective Bacterial Strategy to Escape Host Immune Response and Establish a Chronic Infection. EMBO Mol. Med. 33, 129-141. doi: 10.1002/emmm.201000115

Turner, N. A., Sharma-Kuinkel, B. K., Maskarinec, S. A., Eichenberger, E.M., Shah, P. P., Carugati, M., et al. (2019). Methicillin-Resistant Staphylococcus Aureus: An Overview of Basic and Clinical Research. Nat. Rev. Microbiol. 174, 203-218. doi: 10.1038/s41579-018-0147-4 
von Hoven, G., and Husmann, M. (2019). Staphylococcus Aureus $\alpha$-Toxin's Close Contacts Ensure the Kill. Trends Microbiol. 272, 89-90. doi: 10.1016/ j.tim.2018.11.010

Vozza, E. G., Mulcahy, M. E., and McLoughlin, R. M. (2021). Making the Most of the Host; Targeting the Autophagy Pathway Facilitates Staphylococcus Aureus Intracellular Survival in Neutrophils. Front. Immunol. 12, 667387. doi: 10.3389/fimmu.2021.667387

Wang, H., Zhou, Y., Zhu, Q., Zang, H., Cai, J., Wang, J., et al. (2019). Staphylococcus Aureus Induces Autophagy in Bovine Mammary Epithelial Cells and the Formation of Autophagosomes Facilitates Intracellular Replication of Staph. Aureus. J. Dairy Sci. 1029, 8264-8272. doi: 10.3168/ jds.2019-16414

Watkins, K. E., and Unnikrishnan, M. (2020). Evasion of Host Defenses by Intracellular Staphylococcus Aureus. Adv. Appl. Microbiol. 112, 105-141. doi: 10.1016/bs.aambs.2020.05.001

Weinrick, B., Dunman, P. M., McAleese, F., Murphy, E., Projan, S. J., Fang, Y., et al. (2004). Effect of Mild Acid on Gene Expression in Staphylococcus Aureus. J. Bacteriol 18624, 8407-8423. doi: 10.1128/JB.186.24.8407-8423.2004

Wertheim, H. F., Melles, D. C., Vos, M. C., van Leeuwen, W., van Belkum, A., Verbrugh, H. A., et al. (2005). The Role of Nasal Carriage in Staphylococcus Aureus Infections. Lancet Infect. Dis. 512, 751-762. doi: 10.1016/S1473-3099 (05)70295-4

Wu, H. M., Wang, J., Zhang, B., Fang, L., Xu, K., and Liu, R. Y. (2016). CpG-ODN Promotes Phagocytosis and Autophagy Through JNK/P38 Signal Pathway in Staphylococcus Aureus-Stimulated Macrophage. Life Sci. 161, 51-59. doi: 10.1016/j.lfs.2016.07.016

Xu, F., Li, X., Huang, X., Pan, J., Wang, Y., and Zhou, S. (2020). Development of a $\mathrm{pH}$-Responsive Polymersome Inducing Endoplasmic Reticulum Stress and Autophagy Blockade. Sci. Adv. 631, eabb8725. doi: 10.1126/ sciadv.abb8725

Zang, H., Qian, S., Li, J., Zhou, Y., Zhu, Q., Cui, L., et al. (2020). The Effect of Selenium on the Autophagy of Macrophage Infected by Staphylococcus Aureus. Int. Immunopharmacol. 83, 106406. doi: 10.1016/j.intimp.2020.106406
Zapotoczna, M., Jevnikar, Z., Miajlovic, H., Kos, J., and Foster, T. J. (2013). IronRegulated Surface Determinant B (IsdB) Promotes Staphylococcus Aureus Adherence to and Internalization by non-Phagocytic Human Cells. Cell. Microbiol. 16, 1026-1041. doi: 10.1111/cmi.12097

Zhang, W., Zhuo, S., He, L., Cheng, C., Zhu, B., Lu, Y., et al. (2019). Daphnetin Prevents Methicillin-Resistant Staphylococcus Aureus Infection by Inducing Autophagic Response. Int. Immunopharmacol. 72, 195-203. doi: 10.1016/ j.intimp.2019.04.007

Zhen, X., Lundborg, C. S., Zhang, M., Sun, X., Li, Y., Hu, X., et al. (2020). Clinical and Economic Impact of Methicillin-Resistant Staphylococcus Aureus: A Multicentre Study in China. Sci. Rep. 101, 3900. doi: 10.1038/s41598-02060825-6

Zhu, Y., Li, H., Ding, S., and Wang, Y. (2018). Autophagy Inhibition Promotes Phagocytosis of Macrophage and Protects Mice From Methicillin-Resistant Staphylococcus Aureus Pneumonia. J. Cell. Biochem. 1196, 4808-4814. doi: $10.1002 /$ jcb. 26677

Conflict of Interest: The authors declare that the research was conducted in the absence of any commercial or financial relationships that could be construed as a potential conflict of interest.

Publisher's Note: All claims expressed in this article are solely those of the authors and do not necessarily represent those of their affiliated organizations, or those of the publisher, the editors and the reviewers. Any product that may be evaluated in this article, or claim that may be made by its manufacturer, is not guaranteed or endorsed by the publisher.

Copyright (c) 2021 Wang, Fan and Han. This is an open-access article distributed under the terms of the Creative Commons Attribution License (CC BY). The use, distribution or reproduction in other forums is permitted, provided the original author(s) and the copyright owner(s) are credited and that the original publication in this journal is cited, in accordance with accepted academic practice. No use, distribution or reproduction is permitted which does not comply with these terms. 